\title{
Temporal integration of double electrical pulses
}

\author{
ATSUKI HIGASHIYAMA \\ University of Osaka Prefecture, Osaka, Japan \\ and \\ TAKARA TASHIRO \\ Osaka City University, Osaka, Japan
}

\begin{abstract}
Two experiments investigated temporal integration of double electrical pulses. In Experiment 1, absolute thresholds for double pulses with interstimulus intervals (ISIs) of 0 to $10 \mathrm{msec}$ were determined under three member-pulse durations. In Experiment 2, magnitude estimates for double pulses with ISIs of 0 to $400 \mathrm{msec}$ were obtained at six painful suprathreshold levels. The results, taken together, indicate that (1) partial or no integration takes place at the threshold level, but additive or superadditive integration occurs at the suprathreshold level, and (2) the temporal limit on the integration of double pulses may be longer at the suprathreshold level than at the threshold level, and (3) double pulses at the suprathreshold levels are perceived as most intense for the ISI of about $7 \mathrm{msec}$.
\end{abstract}

Using a single constant-current pulse, a number of studies (Babkoff, Brandeis, \& Bergman, 1975; Girvin et al., 1982; Higashiyama \& Tashiro, 1983; Rollman, 1969, 1974, 1975; Tashiro \& Higashiyama, 1981; Uttal, 1958) have shown that there is an inverse relation between threshold and pulse duration for pulses shorter than $1 \mathrm{msec}$. In mathematical form, this inverse relation can be represented:

$$
\left(I-I_{0}\right) \cdot t^{n}=c,
$$

where $I$ is the threshold current, $t$ is the pulse duration, $n$ is the temporal integration index, and $I_{0}$ and $c$ are constants which depend both on the subject and on the task.

When the integration index $n$ equals unity, doubling $t$ has an effect identical to that of doubling $\left(I-I_{0}\right)$. This perfect temporal integration of electrical current is analogous to Block's law in vision. When $n$ equals zero, current determines sensation, independent of duration. This implies no temporal integration, as is commonly found for durations greater than $10 \mathrm{msec}$. Previous studies (Babkoff et al., 1975; Higashiyama \& Tashiro, 1983) found partial integration to take place for durations between .1 and $1 \mathrm{msec}$, where $n$ is greater than zero but smaller than unity $(n=.4$ to .7$)$. A series of experiments by Larkin and Reilly (1984) suggested that the $n$ value is determined mainly by time constant of electrical pulses: The longer the time constant, the smaller the $n$ value. They accounted for this finding in terms of a resistance-capacitance network at the peripheral level.

In the present study we investigated the psychophysical properties of double electrical pulses at two different

This study was supported in part by a Grant-in-Aid for Scientific Research (\#61510054) provided by the Japanese Ministry of Education, Science, and Culture. A. Higashiyama's mailing address is Psychology Laboratory, University of Osaka Prefecture, Mozu-umemachi, Sakai, Osaka 591, Japan. sensation levels. Experiment 1 compared the effect on integration of single and double pulses at threshold level; it varied the interstimulus interval (ISI), defined as the delay between the offset of the first pulse and the onset of the second one, and the total duration, defined as the sum of the durations of the double pulses. The double pulses were delivered to the same locus. Their threshold $I(t, t)$ was compared with the threshold for single pulses of duration $t$ or $2 t[I(t)$ or $I(2 t)]$.

There were five possible outcomes of Experiment 1. Since, according to Equation 1, the inequality $I(2 t)<I(t)$ should be maintained in all cases in which $n \neq 0$, the only empirical question is where $I(t, t)$ fits into this inequality. (1) It could be that $I(t, t)$ exceeds both $I(t)$ and $I(2 t)$, suggesting that each member of a pulse pair inhibits the effects of the other. (2) A second possibility is that $I(t, t)$ equals $I(t)$ but is larger than $I(2 t)$, indicating that the duration of each member pulse determines the threshold. Hahn (1958) presented evidence for this possibility. Uttal (1959) also showed that when double pulses with 1msec or shorter ISIs were delivered, the effects of the lag pulse disappeared, because the lag pulse fell into the refractory period. (3) It is also possible that $I(t, t)$ is smaller than $I(t)$ but larger than $I(2 t)$. In this case, the threshold is determined by the integration of double pulses, but the integration is not as effective as the integration for a single pulse of duration $2 t$. Rosner (1961) reported that only for ISIs of less than $1 \mathrm{msec}$ were double pulses of duration $2 t$ detected at intensities below the threshold for a single pulse of duration $t$. (4) It is conceivable that threshold depends on the total duration of stimulation. This possibility predicts that $I(t, t)$ could be smaller than $I(t)$ but equal to $I(2 t)$. (5) A final possibility is that $I(t, t)$ is smaller than both $I(t)$ and $I(2 t)$, implying that each member pulse facilitates the effects of the other. To our knowledge, there is no evidence supporting the last two 
Table 1

Five Possible Outcomes Showing the Relation Between Single-Pulse and Double-Pulse Current Thresholds and the Effective Variables or Explanatory Mechanisms Underlying Those Outcomes

\begin{tabular}{ll}
\hline \multicolumn{1}{c}{ Possible Outcome } & \multicolumn{1}{c}{$\begin{array}{c}\text { Effective Variable } \\
\text { or Explanatory Mechanism }\end{array}$} \\
\hline$I(2 t)<I(t)<I(t, t)$ & Mutual inhibition between pulses \\
$I(2 t)<I(t, t)=I(t)$ & Individual pulse duration effective \\
$I(2 t)<I(t, t)<I(t)$ & Partial integration of double pulses \\
$I(2 t)=I(t, t)<I(t)$ & Additive integration of double pulses \\
$I(t, t)<I(2 t)<I(t)$ & Superadditive integration of double pulses \\
\hline Note-See text for definitions of $I(t), I(2 t)$, and $I(t, t)$.
\end{tabular}

possibilities. Table 1 summarizes the five possible outcomes and their underlying effective variables or explanatory interpretations.

Experiment 2 was concerned with the relation of integration to double pulses at the suprathreshold level that produces painful deep pressure. The independent variables were the current and ISI of double pulses. The subject's task was to make verbal estimates of subjective intensity for electrical pulses.

Table 1 is also helpful in predicting the results of Experiment 2 . In this case, the threshold $I$ in Table 1 should be interpreted as the subjective intensity $S$, and the signs of inequality $>$ and $<$ should be $\mathrm{read}\langle$ and $\rangle$, respectively. For example, if superadditive integration of double pulses occurs, it is expected that the relation $S(t, t)>S(2 t)>S(t)$ is obtained from the magnitude estimates for electrical pulses.

The data on temporal integration of double pulses at suprathreshold level, however, are not numerous. The only study is that of Uttal (1959), who demonstrated partial integration of double pulses at a nonpainful suprathreshold level.

\section{EXPERIMENT 1}

\section{Method}

Subjects. The subjects were 5 male undergraduates and 2 male members of the teaching staff. The undergraduate subjects were paid for their participation.

Apparatus. A Nihonkoden electric stimulator provided the basic pulse-forming and -timing circuit. It was used with a Nihonkoden ss-102J isolator, which amplified the basic current pulse delivered by the stimulator and provided a constant-current pulse of controllable amplitude by adjusting a variable resistor in the stimulator. Figure 1 shows tracings of oscilloscopic waveforms that were measured at the output of the isolator: The upper waveform represents a single pulse of $.5 \mathrm{msec}$; the bottom portion represents double pulses of $.5 \mathrm{msec}$ with an ISI of $.5 \mathrm{msec}$. A system comprising an Apple II microcomputer and a Sanwa time regulator determined the time schedule of warning buzzer, foreperiod, and intertrial interval. Pulse shape and duration were calibrated with a Hitachi Type V-302 oscilloscope; pulse amplitude was measured by a Sanwa Electronic Instrument Model $9000 \mathrm{EA}$ digital ammeter.

A pair of rectangular silver electrodes were constructed to fit into a plastic plate (1 cm wide $\times 2.9 \mathrm{~cm}$ long) that was strapped to the underside of the subject's right wrist in the vicinity of the ulnar nerve. The size of each electrode was $1 \times .7 \mathrm{~cm}$; the longer side of an electrode faced that of another electrode, with the inner edges separated laterally by a distance of $1.5 \mathrm{~cm}$.
Procedure. The subject was seated, with his right hand resting on the table. Prior to attachment of the electrodes, the subject's wrist was washed with an alcohol solution. The electrodes were then filled with Keratin electrode paste and applied to the underside of the wrist with the cathode close to the elbow and the anode close to the palm. A trial sequence was started with a warning buzzer and was followed by a single or double pulse to the electrodes after a foreperiod of $2.16,3.47$, or $4.81 \mathrm{sec}$. The foreperiod was randomized for each trial. The intertrial interval was approximately $10 \mathrm{sec}$.

Single or double pulses were delivered from the isolator to the electrodes. The single-pulse durations used were $.1, .2, .6,1.2$, 10 , and 20 msec. For double pulses, the lead and lag pulses were identical in duration and current. The durations of each member pulse were $.1, .6$, and $10 \mathrm{msec}$; the ISIs were $.1, .2, .3, .6,1,2$, 6 , and $10 \mathrm{msec}$. The combination of member-pulse duration and ISI provided 24 different double-pulse stimuli.
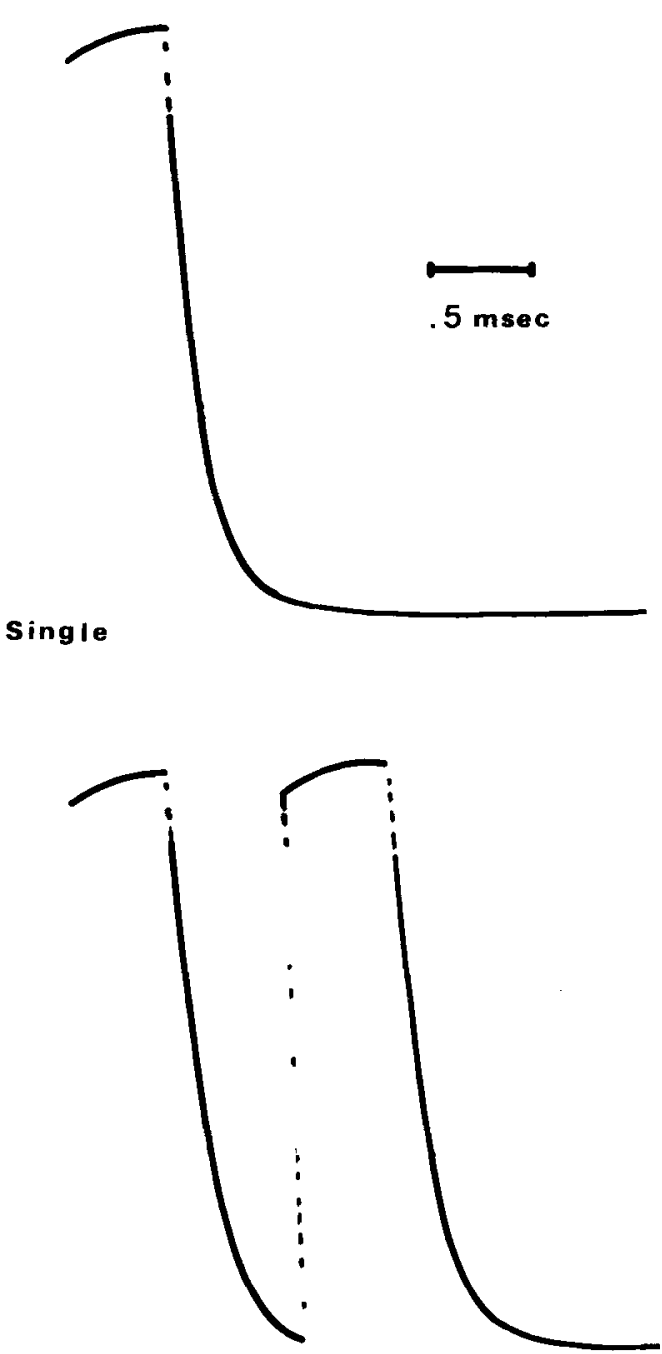

Double

Figure 1. Actual waveforms on an oscilloscope. The upper waveform is that of a single pulse of $.5 \mathrm{msec}$; the lower waveform is that of double pulses of .5 msec each. 
An absolute threshold was determined by a staircase procedure for each pulse condition. Each subject was asked to make more than 32 yes/no judgments of whether current was present on his skin. If the current was detected, it would be decreased by a step (about $.08 \mathrm{~mA}$ ); if not, it would be increased by a step. Single or double pulses were presented on every trial; there were no blank trials.

The experiment consisted of four sessions, one practice session and three main sessions. In each main session, the subject provided eight thresholds for double pulses of a given member-pulse duration $t$ and two thresholds for single pulses of durations $t$ and $2 t$. The presentation order of ISI and pulse duration was determined randomly for each subject.

\section{Results and Discussion}

A threshold was estimated for each pulse condition in accordance with the computational procedure of Dixon and Massey (1957). Figure 2 shows the results of Experiment 1 , with mean threshold plotted as a function of $\log$ ISI. The thresholds for single pulses of duration $t$ are shown on the right side of Figure 2; those for single pulses of duration $2 t$ are shown on the left. It should be noted that the single pulses of durations $t$ and $2 t$ are equivalent to the double pulses of infinite- and 0-msec ISIs, respectively.

A two-way analysis of variance for repeated measures showed that the main effects of ISI $[F(9,54)=3.96$, $p<.01]$ and member pulse $[F(2,12)=10.06, p<.01]$ were significant; the interaction of ISI and member pulse was also significant $[F(18,108)=1.98, p<.05]$. Fur- thermore, the simple main effects of ISI were significant for the member-pulse durations of $.1 \mathrm{msec}[F(9,162)$ $=4.21, p<.001]$ and $.6 \mathrm{msec}[F(9,162)=2.01$, $p<.05]$.

For the member-pulse duration of .1 msec, Tukey HSD tests revealed that the threshold for a single pulse of .2 msec was significantly lower than thresholds for the double pulses with a .3 -msec ISI $(p<.05)$ and a .6-msec ISI $(p<.001)$, and the threshold for a single pulse of $.1 \mathrm{msec}$ was significantly higher than thresholds for the double pulses with a .1-msec ISI $(p<.001)$, a 6 -msec ISI $(p<.05)$, and a 10-msec ISI $(p<.001)$.

For the member-pulse duration of $.6 \mathrm{msec}$, Tukey HSD tests showed that the threshold for a single pulse of $1.2 \mathrm{msec}$ was significantly lower than thresholds for the double pulses with a $.2-\mathrm{msec}$ ISI $(p<.05)$ and a $.6-\mathrm{msec}$ ISI $(p<.05)$, but the threshold for a single pulse of $.6 \mathrm{msec}$ was not significantly different from any threshold of the double pulses employed.

The most important finding of Experiment 1 is that for a member-pulse duration of $.1 \mathrm{msec}$, the relation $I(2 t) \leq I(t, t) \leq I(t)$ held; for a member-pulse duration of .6 msec, the relation $I \leq I(t, t)=I(t)$ held, and for a member-pulse duration of $10 \mathrm{msec}$, the relation $I=I(t, t)$ $=I(t)$ held. This implies that for an extremely short duration of the member pulse, partial integration occurred between double pulses, but for longer duration, detectability was governed by the member pulse.

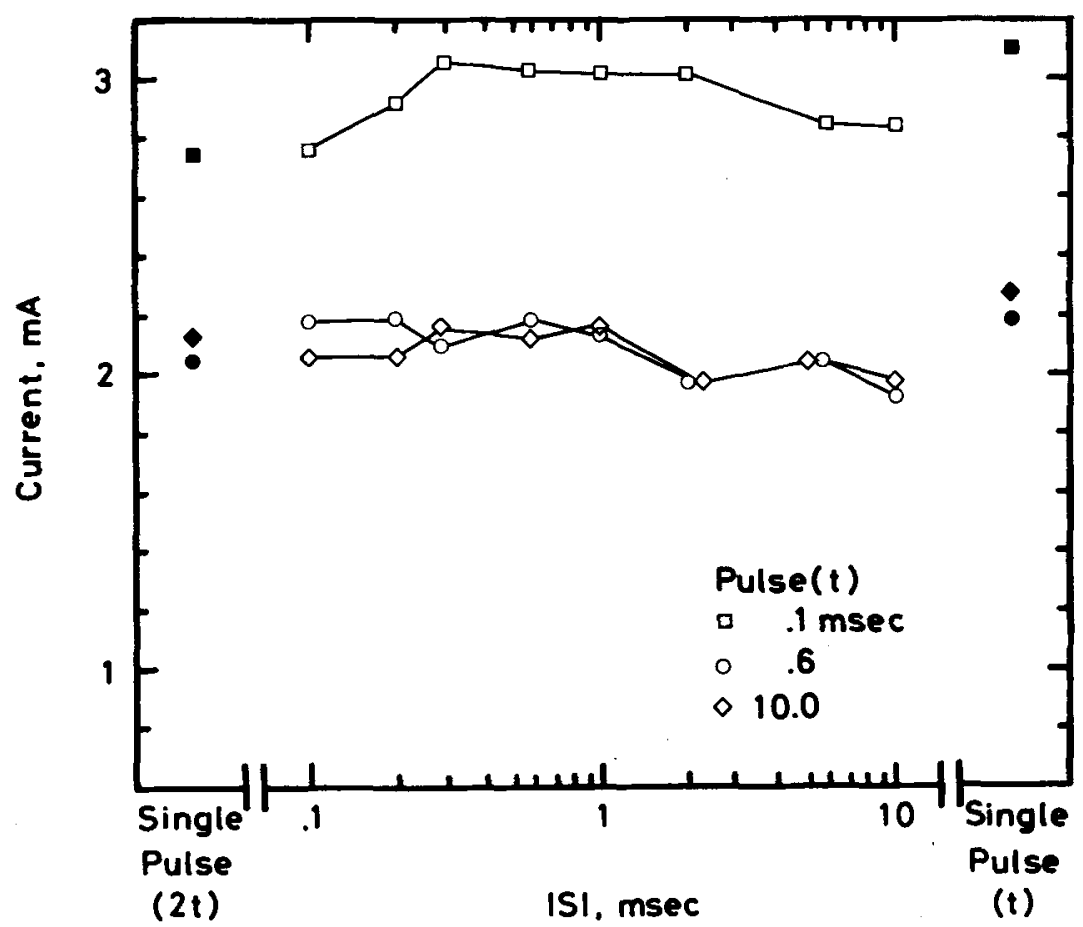

Figure 2. Mean threshold (in $\mathrm{mA}$ ) plotted as a function of $\log$ ISI (in msec). The parameter is the duration of a member pulse. To facilitate a comparison between single and double pulses, the single-pulse data are plotted at the ends of the graph. $t=1 \mathrm{msec}$. 


\section{EXPERIMENT 2}

The purpose of Experiment 2 was to clarify the relation of temporal integration to double pulses at the suprathreshold level. Double pulses of member-pulse duration $t$ were delivered with ISIs varying from .5 to $400 \mathrm{msec}$. The current values used were 2.8 to 3.8 times as intense as the threshold current; these current values often produced painful deep pressure with muscle twitches (Higashiyama \& Tashiro, 1987). The experimenter asked the subjects to estimate the subjective intensities for pulse stimuli. The estimates for double pulses $S(t, t)$ were then compared with those for single pulses $S(t)$ and $S(2 t)$.

\section{Method}

Subjects. The subjects were 20 ( 8 females and 12 males) undergraduates. They were paid for their participation.

Apparatus. The apparatus and electrodes were identical with those of Experiment 1.

Procedure. Each subject was seated, with his/her right hand resting on the table. The method of attaching electrodes and the trial sequence were the same as those of Experiment 1.

Experiment 2 consisted of two parts: measurement of threshold and magnitude estimation. The threshold was determined by the same staircase method as that used in Experiment 1. The subject was asked to make more than 32 yes/no judgments for single pulses of 2 msec duration.

Immediately after the yes/no judgments were completed, the experimenter estimated the threshold current in accordance with the computational procedure of Dixon and Massey (1957), and then determined individually the six current values that were used in the task of magnitude estimation. The current values were 2.8, 3.0, $3.2,3.4,3.6$, and 3.8 times as intense as the threshold.

Single and double pulses were delivered from the isolator to the electrodes. The single-pulse durations used were 1 and $2 \mathrm{msec}$. For double pulses, the duration of each member pulse was $1 \mathrm{msec}$; the ISIs were $.5,1,5,10,20,40,70,100,200$, and $400 \mathrm{msec}$. Thus there were $60(6 \times 10)$ double-pulse stimuli and $12(6 \times 2)$ singlepulse stimuli that resulted from the combination of current and ISI (or single-pulse duration).

Each of the 72 pulse stimuli was presented once, in randomized order, with the restriction that a block include six different current values under a given ISI or single-pulse condition. The experimenter asked the subject to report whether he/she perceived single or separate events for each pulse stimulus. For separate events, the subject reported two numbers, representing the subjective intensity for each member of the pair. For a single event, the subject reported a number that represented the subjective intensity of the fused double pulses or the single pulse. The emphasis of the instructions to the subjects was that the numbers used should reflect the subjective intensities. No modulus or standard was employed.

\section{Results}

The magnitude-estimation data were classified into "single" and "separate" responses. Figure 3 shows the frequency of "separate" responses as a function of $\log$ ISI, with the parameter of ratio of stimulus current to threshold current. The maximum score possible for any stimulus current is 20 . Assuming that the probability of responding "separate" is an ogive of $\log$ ISI, we determined the mean and standard deviation of the underlying Gaussian distribution on the basis of the total scores taken

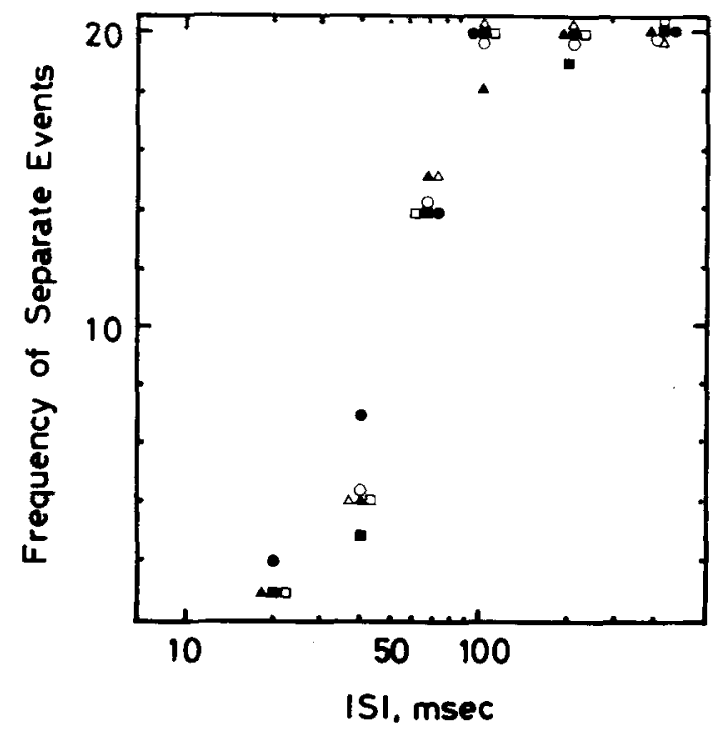

Figure 3. Frequency of separate events as a function of $\log$ ISI (in msec). The parameter is the ratio of stimulus current to threshold current: $\circ, 3.8 ; \Delta, 3.6 ; \square, 3.4 ; \bullet, 3.2 ; \Delta, 3.0 ; \square, 2.8$.

across subjects. The obtained mean and standard deviation were 51.5 and 1.63 , respectively, and the correlation between $\log$ ISI and $z$-score of probability was .958 .

A $t$ test was performed on the magnitude estimates that were given in the instances where the subjects reported two separate responses, for each combination of ISI and current. The results showed that for any combination, the estimates for the lead pulse were not significantly different from those for the lag pulse. Therefore, the subjective intensity for "separate" responses was taken as a mean of the two magnitude estimates.

Figure 4 shows the subjective intensity for double pulses as a function of $\log$ ISI, with the parameter of ratio of stimulus current to threshold current. The magnitude estimates for single pulses of $1 \mathrm{msec}$ duration are shown on the right side of the figure; those for single pulses of 2 msec duration are shown on the left. Each data point is a geometric mean taken across the 20 subjective intensities.

A two-way analysis of variance for repeated measures was performed on the logarithmic transformations of subjective intensities. The results indicated that the main effects of ISI $[F(11,20)=13.10, p<.001]$ and ratio $[F(5,95)=48.80, p<.001]$ were significant, and that the interaction of ISI and ratio was not significant.

The direct method of contrasting the subjective intensities for the single pulses with those for each ISI of double pulses (Keppel, 1973, chap. 20) revealed that the single pulses of $2 \mathrm{msec}$ duration produced significantly smaller estimates than did the double pulses with 5-msec $[F(1,19)=6.12, p<.05], 10-\mathrm{msec}[F(1,19)=12.08$, $p<.01]$, and 20 -msec ISIs $[F(1,19)=7.88, p<.05]$, but yielded significantly larger estimates than did the double pulses with a 400 -msec ISI $[F(1,19)=5.84$, 


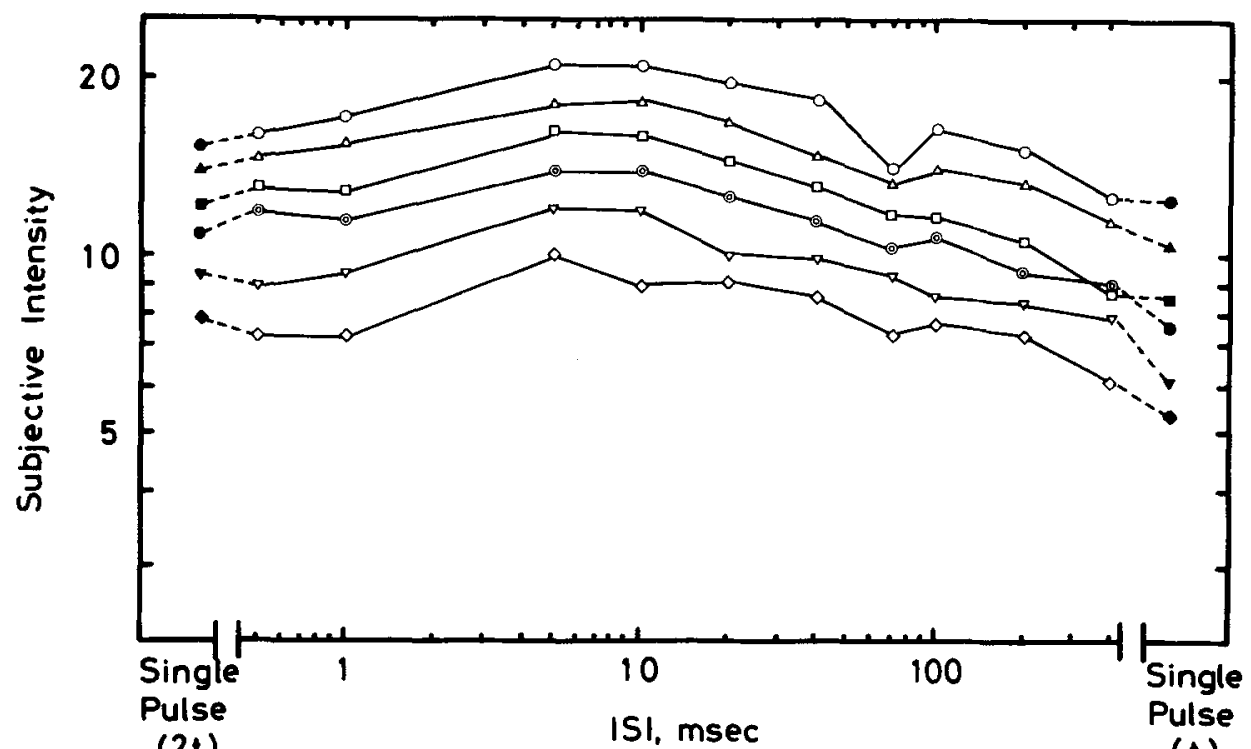

(2t)

ISI, msec

( $t$ )

Figure 4. Log magnitude estimates as a function of $\log$ ISI. Open symbols represent double pulses; filled symbols represent single pulses. The parameter is the ratio of stimulus current to threshold current: 0 , 3.8; $\triangle$, 3.6; $\square, 3.4 ; \odot, 3.2 ; \nabla, 3.0 ; \diamond, 2.8 . t=1$ msec.

$p<.05]$. On the other hand, the single pulses of $1 \mathrm{msec}$ duration produced significantly smaller estimates than did any condition of double pulses [for an ISI of $.5 \mathrm{msec}$, $F(1,19)=24.22, p<.001$; for $1 \mathrm{msec}, F=23.32$, $p<.001$; for $5 \mathrm{msec}, F=36.63, p<.001$; for $10 \mathrm{msec}, F=46.69, p<.001$; for $20 \mathrm{msec}, F=28.89$, $p<.001$; for $40 \mathrm{msec}, F=19.16, p<.001$; for $70 \mathrm{msec}, F=11.21, p<.001$; for $100 \mathrm{msec}, F=$ $15.34, p<.001$; for $200 \mathrm{msec}, F=11.53, p<.01$; for $400 \mathrm{msec}, F=4.64, p<.05]$.

An analysis of trend was applied to log subjective intensities for double pulses as a function of $\log$ ISI. The results showed that the linear $[F(1,19)=42.69$, $p<.001]$ and quadratic $[F(1,19)=23.89, p<.001]$ components were significant and that the variation due to higher order trend components was not significant. As is clear from Figure 4, the significant linear component implies that there was a general negative linear slope, and the significant quadratic component means that there was a rise and then fall to the curve, indicating the presence of a curvilinear trend.

For each ratio of stimulus current to threshold current, the geometric means of subjective intensities for double pulses were fitted by the method of least squares to the quadratic equation

$$
\psi=a+b t+c t^{2},
$$

where $\psi$ stands for log subjective intensity, $t$ for $\log$ ISI, and $a, b$, and $c$ for constants. Table 2 shows the coefficients of the best-fitting quadratic equations and ISIs producing maximum subjective intensity (i.e., $10^{-b / 2 c}$ ).

\section{GENERAL DISCUSSION}

It is clear that in Experiment 1, at the threshold level, partial integration took place only for the double pulses of $.1 \mathrm{msec}$ duration each, and no integration occurred for the double pulses of .6 msec or longer duration each. This finding is consistent with Rosner's (1961) report. In Experiment 2, at the painful suprathreshold level produced by the double pulses of $1 \mathrm{msec}$ duration each, additive or superadditive integration $[S(t, t) \geq S(2 t)>S(t)]$ was obtained for the ISIs of .5 to $200 \mathrm{msec}$, and partial integration $[S(2 t)>S(t, t)>S(t)]$ was found only for the ISI of $400 \mathrm{msec}$.

The results of both experiments taken together suggest that the temporal integration of double pulses occurs for longer ISIs at the suprathreshold level (where, in our experiments, the subject indicated pain intensity) than at the threshold level (where he/she indicated presence or absence of touch). This finding is consistent with the results

Table 2

Parameters of Equation 2, Fitted to the Data of Experiment 2, and the ISI Producing Maximum Subjective Intensity

\begin{tabular}{ccccc}
\hline & \multicolumn{3}{c}{ Parameters } & \\
\cline { 2 - 5 } Ratio & $a$ & $b$ & $c$ & ISI (msec) \\
\hline 2.8 & .897 & .129 & -.065 & 9.82 \\
3.0 & .992 & .105 & -.059 & 7.76 \\
3.2 & 1.095 & .070 & -.052 & 4.71 \\
3.4 & 1.143 & .111 & -.073 & 5.75 \\
3.6 & 1.204 & .081 & -.053 & 5.81 \\
3.8 & 1.247 & .115 & -.068 & 7.01 \\
\hline
\end{tabular}


obtained by Tashiro and Higashiyama (1981), who showed that temporal integration of a single pulse extends to longer durations at the suprathreshold level than near the threshold level. However, this conclusion must be tentative: Since the class of response differs between experiments, we cannot be sure that the changing limit on temporal integration depends on increasing sensation level.

Another finding drawn from our results is that the degree of integration changes depending on level of sensation-partial integration at the threshold level and additive or superadditive integration at the suprathreshold level. To our knowledge, there is no psychophysical study that points out superadditive integration of electrocutaneous pulses. However, a number of physiological studies have provided positive evidence for superadditive integration. Mendell and Wall (1965), Melzack (1973), and Melzack and Wall (1982), for example, indicated that successive intense electrical pulses to small fibers produced a more rapid and long-lasting burst of nerve impulses after each stimulation, whereas successive gentle electrical pulses to large fibers produced a burst of nerve impulses followed by an inhibitory period of nerve activity after each stimulation. However, we could not find the inhibitory effects that would be expected for gentle electrical pulses.

Our third finding is that temporal integration of double pulses is determined not only by the level of sensation, but by the ISI of double pulses. Table 2 and Figure 4 show that for any ratio of stimulus current to threshold current, the subjective intensity of double pulses increased with increasing ISI up to $7 \mathrm{msec}$ (additive to superadditive integration), and decreased progressively beyond that (superadditive to additive integration). These results are in agreement with those of Uttal (1959), who showed that at the nonpainful suprathreshold level, the subjective intensity of double pulses increased monotonically with increasing ISI up to 4 msec.

Our fourth finding is that the minimum ISI for double pulses, being barely distinguished as separate events, was about 50 msec at the suprathreshold level (see Figure 3). This value is very useful in determining the temporal acuity that is defined as the reciprocal of the minimum separation between two stimuli (Piéron, 1952, p. 294). Figure 3 indicates that the temporal acuity for electrical pulses was about $50 \mathrm{msec}$ at the suprathreshold level. It might be argued, however, that for ISIs of 10 to $40 \mathrm{msec}$, distinction between single and separate events was incomplete and the double pulses were perceived as a "jagged" event, instead of a "smooth" event. It is probable that a jagged sensation does occur at some point between single and double responses, but for two reasons, the jagged sensation is not likely to have serious effects on the measurement of temporal acuity. First, it appears that the range of ISIs within which the jagged sensation might have occurred is very narrow, because the slope of the psychometric function (or standard deviation of the underlying Gaussian distribution) in Figure 3 is very steep, indicating that our subjects' responses to double pulses varied from single to separate events with a small increase in ISI. Second, the threshold of $50 \mathrm{msec}$ estimated from Figure 3 is not very different from that obtained by Uttal and Krissoff (1968), who explored the detection threshold of a gap in a train of electrical pulses. They reported that the threshold varied between 30 and $70 \mathrm{msec}$, depending on pulse intensity, interpulse interval, number of pulses, and number of pulses preceeding a gap.

The most important of these findings is that the relation $S(t, t)>S(2 t)$ held for ISIs of 5,10 , and $20 \mathrm{msec}$. We interpret this relation as superadditivity, which may occur at different levels of the somatosensory neural pathways. Another possible explanation that merits discussion is that the subjects made magnitude estimations on the basis of the subjective duration rather than the subjective intensity of fused double pulses. It would be expected from this explanation that the double pulses with ISIs of less than $50 \mathrm{msec}$ ISIs would appear more intense than a single pulse of 2 msec duration. However, the results of Experiment 2 indicated that the double pulses with a $40-\mathrm{msec}$ ISI were perceived as being the same intensity as a single pulse of 2 msec duration, although most subjects perceived these double pulses as a single event.

We obtained the relation $S(t, t)>S(t)$ for the ISIs of 50 to $400 \mathrm{msec}$, at which double pulses were perceived as separate and the members of the pair were perceived as equally intense. This implies that the lead pulse has a retroactive effect on the subjective intensity of the lag pulse, and, at the same time, the lag pulse has a proactive effect on the subjective intensity of the lead pulse. This interpretation is based on the assumption that the interaction of double pulses occurs for fairly long ISIs of 50 to $400 \mathrm{msec}$. This assumption seems to be supported by physiological evidence of pain. Spencer and April (1970) showed that intense electrical stimulation prolonged neural activity of the cat's spinal cord; for example, electrical stimulation delivered for 7 min produced monosynaptic reflex responses of the spinal cord for more than $10 \mathrm{~min}$, and electrical stimulation for $20 \mathrm{~min}$ produced the same responses for $2 \mathrm{~h}$. Melzack (1973, p. 113) observed that brief electrical stimulation of the reticular formation produced long-lasting activity in the medial lemniscus. In this case, stimulus duration was $\mathbf{1 0}$ to $20 \mathrm{sec}$, and the neural response lasted for 5 to $10 \mathrm{~min}$.

Another possible explanation of the relation $S(t, t)>S(t)$ is based on response bias: It may be that, although each member of a double pulse produces the same subjective intensity as the single pulse alone, the subject is likely to judge them as more intense because he/she receives two successive painful shocks from the double pulses. However, it is difficult to interpret the results of Experiment 2 exclusively in terms of response bias, since for the ISIs of less than $50 \mathrm{msec}$, double pulses were perceived as single but as more intense than a single pulse.

Note, finally, that our results should not be accounted for in terms of particular levels of neural activity. At present, available evidence suggests that temporal integra- 
tion for electrical pulses occurs from the peripheral to the central nervous system.

\section{REFERENCES}

Babkoff, H., Brandeis, B., \& Bergman, Y. (1975). Partial integration of single electrocutaneous pulses. Perception \& Psychophysics, 17, 285-292.

Dixon, W. J., \& MASSEY, F. J. (1957). Introduction to statistical analysis. New York: McGraw-Hill.

Girvin, J. P., Marks, L. E., Antunes, J. L., Quest, D. O., O'Keefe, M. D., NING, P., \& DoBELle, W. H. (1982). Electrocutaneous stimulation I. The effects of stimulus parameters on absolute threshold. Perception \& Psychophysics, 32, 524-528.

HAHN, J. F. (1958). Cutaneous vibratory thresholds for square-wave electrical pulses. Science, 127, 879-880.

Higashiyama, A., \& Tashiro, T. (1983). Temporal and spatial integration for electrocutaneous stimulation. Perception \& Psychophysics, 33, 437-442.

Higashiyama, A., \& TAshiro, T. (1987). Magnitude estimates for electrical shocks. Japanese Psychological Research, 29, 81-88.

KePPEL, G. (1973). Design and analysis. Englewood Cliffs, NJ: Prentice-Hall.

LARKIN, W. D., \& REILLY, J. P. (1984). Strength/duration relationships for electrocutaneous sensitivity: Stimulation by capacitive discharges. Perception \& Psychophysics, 36, 68-78.

MelzaCK, R. (1973). The puzzle of pain. New York: Basic Books. Melzack, R., \& Wall, P. D. (1982). The challenge of pain. New York: Penguin Books.

Mendell, L. M., \& WALI, P. D. (1965). Presynaptic hyperpolarization: A role for fine afferent fibers. Journal of Physiology, 172, 274-294.
Pí́ron, H. (1952). The sensations. New Haven, CT: Yale University Press.

Rollman, G. B. (1969). Electrocutaneous stimulation: Psychometric functions and temporal integration. Perception \& Psychophysics, 5, 289-293.

Rollman, G. B. (1974). Electrocutaneous stimulation. In F.A. Geldard (Ed.), Cutaneous communication systems and devices (pp. 3951). Austin, TX: Psychonomic Society.

RollmaN, G. B. (1975). Behavioral assessment of peripheral nerve function. Neurology, 25, 339-342.

ROSNER, B. S. (1961). Neural factors limiting cutaneous spatiotemporal discriminations. In W. A. Rosenblith (Ed.), Sensory communication (pp. 725-737). Cambridge, MA: The MIT Press.

SPENCER, W. A., \& APRI, R. S. (1970). Plastic properties of monosynaptic pathways in mammals. In G. Horn \& R. A. Hinde (Eds.), Short-term changes in neural activity and behaviour (pp. 433474). New York: Cambridge University Press.

Tashiro, T., \& Higashiyama, A. (1981). The perceptual properties of electrocutaneous stimulation: Sensory quality, subjective intensity, and intensity-duration relation. Perception \& Psychophysics, 30, 579-586.

UTtal, W. R. (1958). Cutaneous sensitivity to electrical pulse stimuli. Journal of Comparative \& Physiological Psychology, 51, 549-554.

UTTAL, W. R. (1959). A comparison of neural and psychophysical responses in the somesthetic system. Journal of Comparative \& Physiological Psychology, 52, 485-490.

UTTAL, W. R., \& KrISsofF, M. (1968). Response of the somesthetic system to patterned trains of electrical stimuli. In D. K. Kenshalo (Ed.), The skin senses (pp. 262-303). Springfield, IL: Charles C Thomas.

(Manuscript received March 17, 1987; revision accepted for publication July 17, 1987.) 